\title{
Review of Crowdfunding Regulations across Countries: A Systematic Review Study
}

\author{
Muna M. Alhammad ${ }^{1 *}$, Rawan AlOthman ${ }^{1}$, Chekfoung Tan ${ }^{2}$
}

\author{
${ }^{1}$ College of Business Administration, King Saud University, Riyadh, SAUDI ARABIA \\ ${ }^{2}$ UCL Centre for Systems Engineering, University College London, UK \\ *Corresponding Author: https://orcid.org/0000-0003-2272-757X
}

Citation: Alhammad, M. M., AlOthman, R. and Tan, C. (2021). Review of Crowdfunding Regulations across Countries: A Systematic Review Study. Journal of Information Systems Engineering and Management, 6(4), em0145. https://doi.org/10.21601/jisem/11395

ARTICLE INFO

Received: 23 Apr. 2021

Accepted: 26 Oct. 2021

\begin{abstract}
In recent years, the financial industry is seeing rapid implementation of an increasing number of new technologies. In crowdfunding campaigns, borrowers, potential investors, and platform providers may face some issues in terms of information disclosures, selecting an appropriate threshold, and platform insolvency. However, there is insufficient research to provide a cohesive view of the regulatory framework for crowdfunding platforms. This research offers comparative and comprehensive insights through a systematic literature review (SLR) in analyzing the existing regulatory environment across countries. The results suggest the fundamental regulatory framework in supporting startups, early-stage companies, innovation and entrepreneurs, and balancing investor protections and support for businesses seeking to raise funds, particularly in protecting client's fund, crowdfunding advertising law, fundraising and investment limit, authorization and disclosure obligations. This study contributes to providing a comprehensive understanding of crowdfunding regulatory frameworks and informing governments or policymakers in the future development or reform of crowdfunding regulation frameworks.
\end{abstract}

Keywords: crowdfunding, crowdlending, equity crowdfunding, FinTech, regulatory frameworks, policies, systematic literature review

\section{INTRODUCTION}

Financial Technology (FinTech) has gained tractions in the industry over the years. FinTech is defined as an integration of financial technology that allows customers and businesses to enhance their financial operations in a digital environment without the stiff regulations associated with traditional financial services (Setälä, 2017). This concept eliminates the requirement for physical interaction associated with classical financial services through digitizing the financial services (Aisyah, 2018). Application of FinTech has the power to drive financial services to the end-users efficiently and effectively in different forms such as stock trading, peer-to-peer lending, cryptocurrencies, payment transfers, and crowdfunding (Milian et al., 2019). Crowdfunding platforms have emerged as a successful campaign over the last few years representing a new model of alternative financial intermediation.

Particularly for SMEs, crowdfunding projects are entrepreneurial (Sahaym et al., 2019). Entrepreneurial activities are essential for the economic growth of a nation. Factors such as regulatory business environment will affect the quality and quantity of entrepreneurship (Chowdhury et al.,
2019). Therefore, the regulators who create or update the regulatory framework must take into account all the actors (intermediaries, investors, entrepreneurs) involving in the crowdfunding process. They also must recognize a regime of mutual recognition that the actors of crowdfunding can comply with the rules to enhance innovation and economic development (Kourabas and Ramsay, 2017).

Although crowdfunding regulations are essential to maintain the success of platforms, most of the existing literature is limited to studying country-specific regulations. According to Milian et al. (2019), seeking harmonize international regulation in this area is still in its early stage. Therefore, there is a need for a comprehensive review of crowdfunding regulations across countries to get better insight into the kinds of regulations needed to enhance the safety of using crowdfunding platforms. Hence, this research aims to provide comprehensive insight into the crowdfunding regulations across different countries worldwide by conducting a systematic literature review. This paper will examine the current trends of crowdfunding regulations and identify the common essential regulations to help maintaining the success of a crowdfunding platform while ensuring its safety. This study enables lawmakers, legislation practitioners, 
and government agencies to make informed decisions regarding crowdfunding practices and transactions.

The remainder of this paper is structured into six sections: Section Related Work presents related work. Section Review Method describes the review methodology for conducting this review, followed by the research results and discussions in Section Results: Regulation Themes and Section Discussions. Section Conclusion and Future Works concludes the study with research contributions and future work.

\section{RELATED WORK}

Crowdfunding is a money-raising method for individuals or small organisations such as small, medium enterprises (SMEs) and start-ups using the Internet, which is also known as a funding portal (Gabison, 2015; Lukstinšs, 2017). It involves three actors, namely funding portals (intermediaries), contributors (funders/investors) and crowdfunding campaign creators (fund seekers/entrepreneurs) (Hofmann, 2018). As the first action in the crowdfunding process, crowdfunding campaign creators, who are seeking funds from investors, must register and create an account on a crowdfunding portal. Fund seekers should provide their identity information or prior information on the funding portal during this registration process (Estrin et al., 2016; Lukstiňš, 2017). After registration, the crowdfunding project creator, the borrower, entrepreneur or SMEs, will start a crowdfunding campaign on the platform by creating a project that seeks funds. Then, the platform providers will evaluate and cross-check the information provided by the fund seekers about the business idea, experience, requested funding amount, and validate fund seekers' personal information. Meanwhile, platform providers also evaluate the security and repayment method as this is an important aspect in crowdfunding to make sure all investors are paid back at the end of the campaign (Lukstinšs, 2017). The platform management will approve projects that are suitable for financing. Usually, the management will request the borrower to sign a loan agreement when they approve a project. Finally, the project will be published and start collecting fundraising money on the funding portal (Lukstinšs, 2017).

There are four typical types of crowdfunding: donationbased, reward-based, lending-based and equity-based crowdfunding. Donation and reward-based crowdfunding are classified as non-financial return crowdfunding while the rest of crowdfunding types, including lending-based and equitybased, are classified as crowdfunding with a financial return (Gabison, 2015; Herrera, 2016; Lee and Kim, 2015; Lukstiņš, 2017). Donation-based crowdfunding platforms such as GoFundMe, 1\%Club and Act4Greece remain a major source of crowdfunding compared to other types of crowdfunding campaigns. They are often called philanthropic campaigns, not-for-profit campaigns or sponsorship campaigns; hence, contributors do not receive any returns other than publicity (Gabison, 2015; Spanos, 2018). In contrast, in reward-based campaigns, contributors will usually obtain a product or service in return. Common examples of reward-based crowdfunding platforms are Kickstarter in the US, Verkami in Spain, Crowdculture in Sweden, and ZEQUS in the UK.
Lending-based crowdfunding has emerged as an alternative method to bank loans, where lenders provide their money to borrowers and expect a return in the form of capital and interest. Examples of lending-based platforms are Lending Club in the US, Zopa in the United Kingdom, and LeihDeinerStadtGeld in Germany. Equity-crowdfunding is an Internet-based limited initial public offering (IPO), where contributors receive the rewards as forms of shares (Gabison, 2015). EquityNet, Symbid and Socioinversores are examples of equity-based crowdfunding in the US, Netherlands, and Spain, respectively. Moreover, there are several platforms offering equity-based intermediaries services such as Seedrs from the UK and MyMicroInvest in Belgium.

Although crowdfunding has many success stories, it also has several drawbacks as it is in the beginning stage and is highly unpredictable (Ng and Kwok, 2017). Many researchers indicated that the risks associated with crowdfunding are similar to the risks associated with any different financial sectors, such as investment and securities activities and payment services. However, as crowdfunding is operated online, it is also significantly exposed to the vulnerability of Internet-related threats and other related technological risks such as cyber-attacks (Ng and Kwok, 2017). Additionally, many crowdfunding platforms operate across borders and widen the legal complexities (Kabai, 2017). Crowdfunding, as a business model, is considered a vulnerable activity as investor's higher returns are linked to less solvent borrowers. Financing through crowdfunding means a high risk of loss as most of the business are start-ups or early-stage businesses that regularly lack sufficient collateral, as well as a guarantee of repayment in case of a borrower's default (Monés, 2018).

Moreover, Wroldsen (2016) argued that turning the general public (unaccredited investors) into a miniature venture capitalist may be associated with inherent risks. The general public usually has difficulty making the right decision about investing in start-up companies that have the most probability to fail in the early stage. If the platform providers conduct insufficient and inefficient due diligence on the borrower's creditworthiness, investors may end up in liquidity risk. This scenario is due to the fact that the investor's decision-making in selecting borrower profiles is primarily based on the information offered by the platform providers. Investors can avoid insolvent borrowers or mitigate blur transparency if the platform provides efficient due diligence (Hofmann, 2018; Monés, 2018). Investors may also be exposed to a risk of platform insolvency or platform failure. In this case, investors are at risk of not being repaid by borrowers as the funding portals are the one responsible for getting the loan back (Monés, 2018).

\section{REVIEW METHOD}

The systematic literature review (SLR) method is an unbiased approach for data gathering with a systematic way of identifying, evaluating and interpreting data using predefined research queries that are relevant to a phenomenon of interest (Kitchenham, 2004). Moreover, SLR helps to identify gaps in the existing literature for further investigation and provides a deeper understanding of a new phenomenon 
Table 1. Inclusion and exclusion criteria

\begin{tabular}{|c|c|c|}
\hline $\begin{array}{l}\text { Inclusion Criteria } \\
\end{array}$ & \multicolumn{2}{|c|}{ Exclusion Criteria } \\
\hline Studies that discuss the topic of crowdfunding specifically. & \multicolumn{2}{|c|}{ Studies published in languages other than English. } \\
\hline Studies that address research questions directly or indirectly. & \multicolumn{2}{|c|}{ Duplicate, incomplete studies, news, and trade journals. } \\
\hline $\begin{array}{c}\text { Studies discuss crowdfunding regulations, policies, laws, } \\
\text { legislation, and disclosure laws. }\end{array}$ & \multicolumn{2}{|c|}{$\begin{array}{l}\text { Studies which were not relevant to crowdfunding regulations such as factors } \\
\text { impacting users' behavior, Fintech regulations in general, and IPOs. }\end{array}$} \\
\hline Table 2. Overview of data sources & & \\
\hline Data sources & Studies found & Selected study \\
\hline Google Scholar & 889 & 10 \\
\hline IEEE Xplore & 58 & 1 \\
\hline Springer & 73 & 3 \\
\hline HeinOnline & 27 & 5 \\
\hline SSRN's eLibrary & 220 & 7 \\
\hline Science Direct & 5 & 2 \\
\hline Total & 1272 & 28 \\
\hline
\end{tabular}

Table 3. Quality assessment results

\begin{tabular}{ccc}
\hline Source & Score & $\begin{array}{c}\text { Number of } \\
\text { papers }\end{array}$ \\
\hline $\begin{array}{cc}\text { (Rossi et al., 2019), (Gabison, 2015), (Havrylchyk, 2018), (Maguire and Delahunt, 2017), (Lee and Kim, 2015), (Hofmann, } \\
\text { 2018), (Jagtiani and Lemieux, 2018), (Rau, 2018), (Monés, 2018), (Herrera, 2016), (Kourabas and Ramsay, 2017), (Fenwick } \\
\text { et al., 2017), (Ahlstrom et al., 2018), (Ibrahim, 2016), (Kourabas and Ramsay, 2017), (Mills, 2016), (Spanos, 2018), } \\
\text { (Mamonov and Malaga, 2018), (Matthew, 2017), (Estrin et al., 2016), (Lukstiňš, 2017), (Huang and Zhao, 2017) }\end{array}$ & 4 \\
\hline (Ahern, 2018), (Chang, 2020) & 3.5 \\
\hline (Groshoff et al., 2015), (Rui-Teng Hsueh et al., 2016), (Macchiavello, 2018), (Frydrych and Kinder, 2015) & 3 \\
\hline
\end{tabular}

(Kitchenham, 2007). The SLR protocol identified by Kitchenham (2007) was adopted in this research; the following sub-sections explain the review details.

\section{Search Strategy}

The study search strategy started by utilizing online digital databases, mainly Google Scholar, IEEE Xplore, Springer, HeinOnline, SSRN's eLibrary, and ScienceDirect. Google Scholar was mainly chosen because it includes common academic databases like Emerald and other financial databases. For an effective query, a list of search strings was identified to search for the relevant studies. The search string used in this SLR is given below: ("crowdsourcing" OR "crowd lending” OR "Crowdfunding” OR “online lending”) AND ("Fintech" OR "finance technology" OR "financial information systems”). We set the search period from the year 2000 to the present in order to guarantee enough timeframe coverage of literature.

Inclusion and exclusion criteria were applied in this research context. These criteria ensure that all selected studies were appropriate and relevant. Although it was sufficient to determine a candidate study as the selected study by merely reading the title from different sources, the title, abstract and keywords of each selected study were considered to optimize selection precision. Table 1 summarises the inclusion and exclusion criteria and Table 2 depicts the data sources and selected studies.

\section{Quality Assessment}

Performing a quality assessment screening is important after finishing the process of inclusion and exclusion criteria (Al-Emran et al., 2018). Therefore, to evaluate the quality of the initially selected studies, the researchers formulated four quality assessment criteria. The developed QA criteria are as follow: 1) Are the objectives of the research outlined clearly? 2) Is the paper studying crowdfunding in particular? 3) Is the paper focusing on crowdfunding regulations related to platforms, investors, and borrowers? 4) Are the results and findings of the research clearly reported? Each of the selected studies was examined on the basis of formulated QA criteria and was ranked as having high, medium, or low quality. Each indicator will be scored as follows: 1 for a fully answered question, 0.5 for a partially answered question, and 0 for an unanswered question. Table 3 illustrates the outcome of the quality assessment, which examined all the twenty-eight selected studies against QA criteria. Notably, all the papers qualified, with a majority of the papers receiving the highest score, which were then forwarded for the analysis shown in Table 3.

\section{Data Extraction and Analysis}

In this step, the data extraction form was developed, and the data was extracted and recorded from the twenty-eight selected studies that was refined from the previous step. The data extraction and syntheses process started with deep scanning to extract the relevant data that answers the formulated research questions. Thematic analysis, which is defined as the process of identifying patterns or common themes within qualitative data (Maguire and Delahunt, 2017), was used to extract the data. The extracted data were recorded using Microsoft Excel spreadsheets. To ensure the validity of the extracted data, the authors analyzed all of the papers and their analysis were then compared and calibrated to ensure that internal consistency was maintained. Any conflict in the analysis was solved and agreed upon before proceeding with the next stage of the analysis. In the next stage, the data were 
Table 4. Summary of selected studies

\begin{tabular}{|c|c|c|c|c|c|c|c|c|c|c|c|c|c|c|c|c|c|c|c|c|c|c|c|c|c|c|c|c|}
\hline Source & 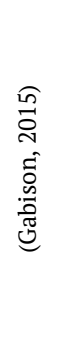 & 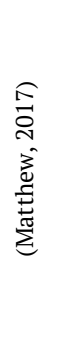 & 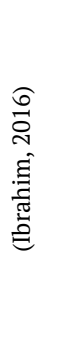 & 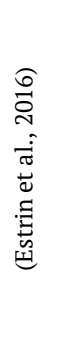 & 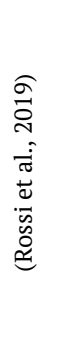 & 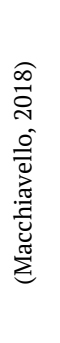 & 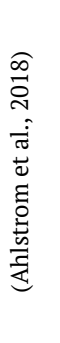 & 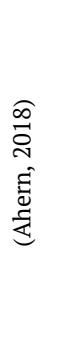 & 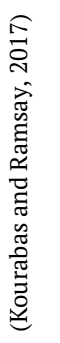 & 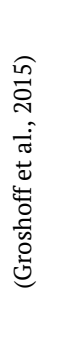 & 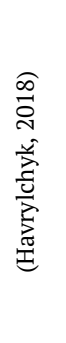 & 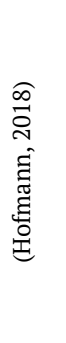 & 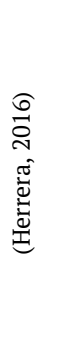 & 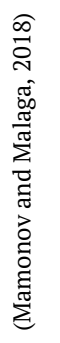 & 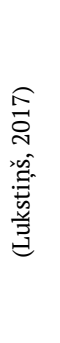 & 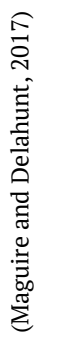 & 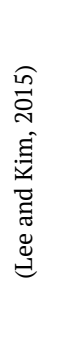 & 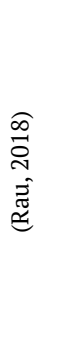 & 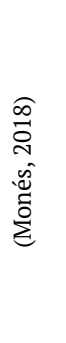 & 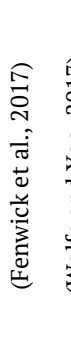 & 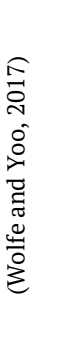 & 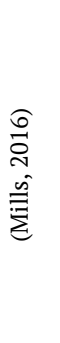 & 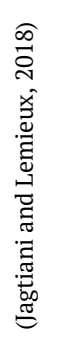 & 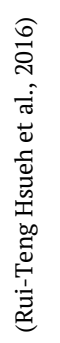 & 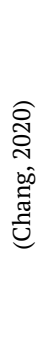 & 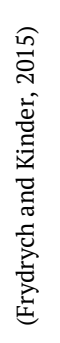 & 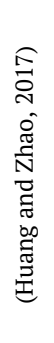 & 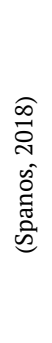 \\
\hline $\begin{array}{l}\text { Methods: } \\
\text { Case Study }\end{array}$ & $\mathrm{x}$ & $\mathrm{x}$ & $\mathrm{x}$ & $\mathrm{X}$ & & $\mathrm{x}$ & $\mathrm{x}$ & & $\mathrm{x}$ & & & $\mathrm{x}$ & $\mathrm{x}$ & $\mathrm{X}$ & & $\mathrm{x}$ & $\mathrm{x}$ & $\mathrm{x}$ & $\mathrm{x}$ & $\mathrm{x}$ & $\mathrm{x}$ & $\mathrm{X}$ & & $\mathrm{x}$ & & & & \\
\hline \multicolumn{29}{|l|}{$\begin{array}{c}\text { Action } \\
\text { Research }\end{array}$} \\
\hline \multicolumn{29}{|l|}{ Survey } \\
\hline \multicolumn{29}{|l|}{$\begin{array}{c}\text { Systematic } \\
\text { Literature } \\
\text { Review }\end{array}$} \\
\hline \multicolumn{29}{|l|}{$\begin{array}{l}\text { Systematic } \\
\text { Mapping }\end{array}$} \\
\hline $\begin{array}{l}\text { Exploratory } \\
\text { Research }\end{array}$ & & & & $\mathrm{x}$ & & & $\mathrm{x}$ & & & & & & & & $\mathrm{x}$ & & & & & & & & & $\mathrm{x}$ & $\mathrm{X}$ & $\mathrm{x}$ & $\mathrm{x}$ & \\
\hline \multicolumn{29}{|l|}{$\begin{array}{c}\text { Controlled } \\
\text { experiments }\end{array}$} \\
\hline $\begin{array}{c}\text { Literature } \\
\text { Survey }\end{array}$ & $\mathrm{x}$ & $\mathrm{x}$ & $\mathrm{x}$ & & $\mathrm{x}$ & $\mathrm{x}$ & & $\mathrm{x}$ & & $\mathrm{x}$ & $\mathrm{X}$ & & $\mathrm{x}$ & & & & & & $\mathrm{x}$ & & $\mathrm{x}$ & $\mathrm{x}$ & $\mathrm{x}$ & & & & & $\mathrm{X}$ \\
\hline \multicolumn{29}{|l|}{ Unclear } \\
\hline $\begin{array}{c}\text { Data Analysis: } \\
\text { Qualitative }\end{array}$ & & $\mathrm{x}$ & $\mathrm{x}$ & $\mathrm{x}$ & $\mathrm{x}$ & $\mathrm{x}$ & $\mathrm{x}$ & $\mathrm{X}$ & $\mathrm{x}$ & $\mathrm{x}$ & $\mathrm{x}$ & & $\mathrm{x}$ & $\mathrm{X}$ & $\mathrm{x}$ & & $\mathrm{x}$ & $\mathrm{x}$ & $\mathrm{x}$ & $\mathrm{x}$ & $\mathrm{x}$ & $\mathrm{X}$ & $\mathrm{x}$ & $\mathrm{x}$ & & & & \\
\hline Quantitative & & & & & & & & & & & & & & & & & & & & & & & & & $\mathrm{X}$ & $\mathrm{X}$ & $\mathrm{X}$ & $\mathrm{X}$ \\
\hline Mixed & $\mathrm{X}$ & & & & & & & & & & & $\mathrm{X}$ & & & & $\mathrm{X}$ & & & & & & & & & & & & \\
\hline \multicolumn{29}{|l|}{ Unclear } \\
\hline $\begin{array}{l}\text { Study setting: } \\
\text { Academic }\end{array}$ & $\mathrm{x}$ & $\mathrm{X}$ & $\mathrm{x}$ & $\mathrm{X}$ & $\mathrm{X}$ & $\mathrm{x}$ & & $\mathrm{x}$ & & & $\mathrm{x}$ & $\mathrm{x}$ & & $\mathrm{x}$ & & $\mathrm{x}$ & $\mathrm{X}$ & $\mathrm{x}$ & $\mathrm{x}$ & $\mathrm{x}$ & & $\mathrm{X}$ & $\mathrm{X}$ & $\mathrm{X}$ & $\mathrm{x}$ & & $\mathrm{x}$ & $\mathrm{x}$ \\
\hline $\begin{array}{l}\text { Industry } \\
\end{array}$ & & & & & & & $\mathrm{X}$ & & $\mathrm{X}$ & $\mathrm{X}$ & & & $\mathrm{X}$ & & $\mathrm{X}$ & & & & & & & & & & & $\mathrm{X}$ & & \\
\hline Unclear & & & & & & & & & & & & & & & & & & & & & $\mathrm{X}$ & & & & & & & \\
\hline \multicolumn{29}{|l|}{$\begin{array}{c}\text { Data } \\
\text { Collection } \\
\text { Approach: } \\
\text { Observation }\end{array}$} \\
\hline \multicolumn{29}{|l|}{ Training } \\
\hline \multicolumn{29}{|l|}{ Workshop } \\
\hline Questionnaire & & & & & & & & & & & & & & & & & & & & & $\mathrm{X}$ & & & & & & & \\
\hline Interviews & & & & $\mathrm{X}$ & & & & & $\mathrm{X}$ & & & & & & & $\mathrm{X}$ & & & & & & & & & & & & \\
\hline $\begin{array}{c}\text { Archival } \\
\text { Record }\end{array}$ & $\mathrm{X}$ & $\mathrm{x}$ & $\mathrm{X}$ & $\mathrm{x}$ & $\mathrm{X}$ & $\mathrm{x}$ & $\mathrm{x}$ & $\mathrm{X}$ & & $\mathrm{X}$ & $\mathrm{x}$ & $\mathrm{x}$ & $\mathrm{X}$ & $\mathrm{x}$ & $\mathrm{x}$ & $\mathrm{x}$ & $\mathrm{x}$ & $\mathrm{x}$ & $\mathrm{x}$ & $\mathrm{x}$ & $\mathrm{x}$ & $\mathrm{x}$ & $\mathrm{X}$ & $\mathrm{X}$ & $\mathrm{x}$ & $\mathrm{x}$ & $\mathrm{x}$ & $\mathrm{x}$ \\
\hline $\begin{array}{c}\text { Visit } \\
\text { experiment }\end{array}$ & & & & & & & & & & & & & & & & & & & & & & & & & & & & \\
\hline
\end{tabular}

cross checked using countries crowdfunding regulatory acts to ensure the validity of the identified regulations.

Table 4 presents a summary of the selected studies with details of the research method, data analysis, study setting, and data collection used in each particular study. The outcomes of the thematic analysis that can help in addressing the research questions will be discussed in detail in the following section.

\section{RESULTS: REGULATION THEMES}

This review resulted in identifying the regulatory environment in twenty-six countries. These were the only countries found in the literature review. The countries include US, Mexico, Canada, UK, EU, Spain, Italy, Austria, Belgium,
Estonian, Finland, France, Germany, Portugal, Latvia, Greece, Norway, Netherland, Singapore, China, Hong Kong, Thailand, Taiwan, Australia, Malaysia and New Zealand. The key regulation themes include authorization, fundraising and investment limits, disclosures obligations, crowdfunding advertising law, capital requirements policies, protection of client funds, and platform-specific regulation. Table 5 summarizes the reference of countries and their applicable crowdfunding regulation requirements.

\section{Authorization}

Crowdfunding platforms operators must be authorized in order to play a role as an intermediary in securities investments. The authorization process starts with reviewing the documents submitted by the platform operator meeting a set of requirements imposed by the financial authority to 
Table 5. Countries and their crowdfunding regulation requirements

\begin{tabular}{|c|c|c|c|c|c|c|c|c|c|c|c|c|c|c|c|c|c|c|c|c|c|c|c|c|c|c|c|}
\hline \multicolumn{2}{|c|}{ نे } & s & 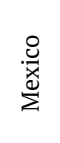 & 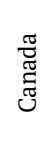 & 弚 & 畕 & $\begin{array}{l}\text { 芯 } \\
\text { के }\end{array}$ & $\frac{\vec{\lambda}}{\underline{\Xi}}$ & 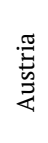 & 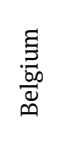 & 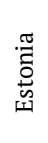 & 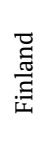 & 芯 & 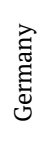 & $\begin{array}{l}\overrightarrow{\widetilde{J}} \\
0 \\
0 \\
0 \\
0 \\
0\end{array}$ & 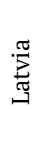 & 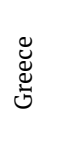 & 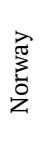 & 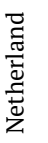 & $\begin{array}{l}\frac{\pi}{0} \\
\frac{\pi}{\pi} \\
\frac{\pi}{2}\end{array}$ & 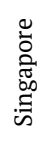 & 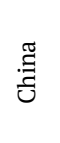 & 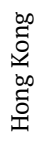 & 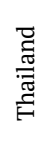 & $\underset{\substack{\pi\\
}}{\stackrel{\Xi}{\pi}}$ & 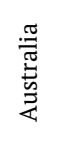 & 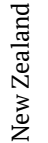 \\
\hline \multicolumn{2}{|c|}{ Authorization } & $\sqrt{\sqrt{ } 1,2}$ & $\sqrt{ }$ & $\sqrt{ }^{1}$ & $\sqrt{ }$ & $\sqrt{ }{ }^{2}$ & $\sqrt{V}$ & $\sqrt{ }{ }^{2}$ & $\sqrt{ }$ & $\sqrt{ }$ & $\sqrt{ }$ & $\sqrt{ }$ & $\sqrt{V^{2}}$ & $\sqrt{\sqrt{ }^{2}}$ & $\sqrt{2}$ & $\sqrt{ }$ & $\sqrt{ }$ & $\sqrt{ }$ & $\mathrm{V}$ & $\sqrt{ }$ & $\sqrt{\sqrt{1,2}}$ & $\sqrt{ }$ & $\sqrt{ }$ & $\sqrt{ }^{1}$ & $\sqrt{ }$ & $\sqrt{ }{ }^{1}$ & $\sqrt{ }$ \\
\hline \multirow{2}{*}{$\begin{array}{c}\text { Fundraising } \\
\text { limits over } 12 \\
\text { months } \\
\end{array}$} & Investors & $\sqrt{ }$ & & $\sqrt{ }$ & $\sqrt{ }$ & & $\sqrt{ }$ & $\sqrt{ }$ & $\sqrt{ }$ & $\sqrt{ }$ & & $\sqrt{ }$ & $\sqrt{ }$ & $\sqrt{ }$ & $\sqrt{ }$ & $\sqrt{ }$ & $\sqrt{ }$ & & $\sqrt{ }$ & $\sqrt{ }$ & & $\sqrt{ }$ & & $\sqrt{ }$ & $\sqrt{ }$ & $\sqrt{ }$ & \\
\hline & Companies & $\sqrt{ }$ & & $\sqrt{ }$ & $\sqrt{ }$ & $\sqrt{ }$ & $\sqrt{ }$ & $\sqrt{ }$ & $\sqrt{ }$ & & & & $\checkmark$ & $\sqrt{ }$ & $\sqrt{ }$ & & $\sqrt{ }$ & & $\checkmark$ & $\sqrt{ }$ & $\sqrt{ }$ & $\sqrt{ }$ & & $\checkmark$ & $\sqrt{ }$ & $\sqrt{ }$ & $\checkmark$ \\
\hline \multirow{4}{*}{$\begin{array}{l}\text { Disclosures } \\
\text { obligations }\end{array}$} & Financial & $\sqrt{3}$ & & $\sqrt{ }$ & $\sqrt{ }$ & $\sqrt{ }$ & & & $\sqrt{ }$ & & $\sqrt{3}$ & & & & $\sqrt{ }$ & & & & & $\sqrt{ }$ & $\sqrt{ }$ & & & & & $\sqrt{ }$ & $\sqrt{ }$ \\
\hline & Annual & $\sqrt{ }^{2}$ & & $\sqrt{ }$ & & & $\sqrt{ }$ & & $\sqrt{ }$ & & & & $\sqrt{ }$ & & $\sqrt{ }$ & & & & & & & & & & & & \\
\hline & Prospectus & $\sqrt{ }$ & & $\sqrt{\sqrt{ }^{1}}$ & $\sqrt{ }{ }^{1}$ & $\sqrt{1,3}$ & & $\sqrt{ }^{1}$ & $\sqrt{ }$ & $\sqrt{1,3}$ & $\sqrt{ }$ & $\sqrt{\sqrt{1}}$ & $\sqrt{{ }^{1}}$ & $\sqrt{\sqrt{ }^{1}}$ & & $\sqrt{3}$ & & & & & $\sqrt{V^{1}}$ & & & $\sqrt{{ }^{1}}$ & & $\sqrt{ }{ }^{2}$ & \\
\hline & $\begin{array}{c}\text { Other form } \\
\text { of } \\
\text { disclosures }\end{array}$ & $\sqrt{ }$ & $\sqrt{ }$ & $\checkmark$ & $\checkmark$ & $\sqrt{ }$ & $\checkmark$ & & $\sqrt{ }$ & $\checkmark$ & $\checkmark$ & $\checkmark$ & $\checkmark$ & $\sqrt{ }$ & $\sqrt{ }$ & & & & & $\sqrt{ }$ & $\sqrt{ }$ & & & $\sqrt{ }$ & & $\sqrt{ }^{1}$ & $\sqrt{ }$ \\
\hline \multirow{2}{*}{\multicolumn{2}{|c|}{$\begin{array}{c}\text { CF advertising law } \\
\text { Capital Requirements } \\
\text { Policies }\end{array}$}} & $\sqrt{3}$ & & $\sqrt{ }$ & $\sqrt{3}$ & & $\sqrt{ }$ & & & & & & & & & & & & & $\sqrt{ }$ & $\sqrt{ }$ & & & $\sqrt{3}$ & & $\sqrt{ }$ & \\
\hline & & & & & $\checkmark$ & $\checkmark$ & $\checkmark$ & & $\sqrt{ }$ & $\checkmark$ & & $\checkmark$ & $\checkmark$ & $\sqrt{1,2}^{1,2}$ & $\checkmark$ & $\checkmark$ & & & & & $\checkmark$ & & & & $\sqrt{ }$ & & \\
\hline \multirow{2}{*}{\multicolumn{2}{|c|}{ Protection of Clients funds }} & & $\sqrt{ }$ & & $\sqrt{ }$ & $\sqrt{ }$ & & & $\checkmark$ & $\checkmark$ & & $\sqrt{ }$ & $\sqrt{ }$ & & & $\sqrt{ }$ & & & & & $\sqrt{ }$ & & & $\sqrt{ }$ & & & \\
\hline & & $\sqrt{ }$ & & & & & & & & & & & & & & & $\sqrt{ }$ & & & & & & & & & $\sqrt{ }$ & \\
\hline
\end{tabular}

(1) With exemption, (2) With extra requirements, (3) With some conditions

Table 6. Authorization requirements

\begin{tabular}{|c|c|c|}
\hline $\begin{array}{l}\text { Region / } \\
\text { Countries }\end{array}$ & Exemption of registration requirements & Additional requirements \\
\hline EU & & \multirow{2}{*}{$\begin{array}{c}\text { Private investigator (PI) license (Havrylchyk, 2018; Hofmann, } \\
\text { 2018) }\end{array}$} \\
\hline Portugal & & \\
\hline US & $\begin{array}{l}\text {-Issuer offered to the public not exceed 5M } \\
\text { - Federal registration exempt from state-level registration }\end{array}$ & $\begin{array}{c}\text { Indiana: collection agency license Mississippi: loan brokering } \\
\text { license (Wolfe and Yoo, 2017) }\end{array}$ \\
\hline Italy & & Professional insurance (Havrylchyk, 2018; Hofmann, 2018) \\
\hline Germany & & Professional liability insurance (Havrylchyk, 2018; Hofmann, 2018) \\
\hline France & & Civil and Professional liability insurance \\
\hline Italy & & Professional insurance (Havrylchyk, 2018; Hofmann, 2018) \\
\hline Singapore & $\begin{array}{l}\text { If lending to business entities or accredited investors } \\
\text { (Hofmann, 2018) }\end{array}$ & $\begin{array}{l}\text { Moneylender's license if lending to non-accredited (Havrylchyk, } \\
\text { 2018; Hofmann, 2018) }\end{array}$ \\
\hline Thailand & $\begin{array}{l}\text { If offered to retail investors THB 20M or less over } 12 \text { months } \\
\text { or not exceeding THB 40M in total or not exceeding THB 50K } \\
\text { for each issuer (Kourabas and Ramsay, 2017) }\end{array}$ & \\
\hline Australia & $\begin{array}{c}\text { Not more than AUD 5M for any business (Gabison, 2015; } \\
\text { Kourabas and Ramsay, 2017) }\end{array}$ & \\
\hline Canada & Start-up companies (Kourabas and Ramsay, 2017) & \\
\hline
\end{tabular}

achieve the minimum threshold to be authorized. Threshold conditions include the current legal status of the company, its non-financial and financial resources, organizational structure, operational or nearly operational situation, and corporate profile (Herrera, 2016). Table 6 shows a summary of authorization regulations.

In all twenty-six countries, the regulation on authorization and licensing process generally require a prospective crowdfunding platform operator to operate under a license through registration with a regulatory authority of the respective jurisdiction. The underlying reasons behind the authorization process for crowdfunding platform operators are to ensure that the platform operators comply with legal obligations, reflect high standards of commercial integrity, and prevent fraudulent activities such as money-laundering, and these rules should comply with organizational requirements (Herrera, 2016; Hofmann, 2018; Kourabas and Ramsay, 2017). Concerning authorization requirements, having additional obligations, e.g., professional liability insurance, could assist in providing a great deal of protection for investors in the event of the platform owner insolvency.
In addition, some countries stated that the issuer could qualify for an exemption from the authorization requirement if the total amount offered to the public does not exceed the minimum threshold value. For instance, the US offers an authorization exemption if the amount offered to the public is less than USD 5 million. Also, the US Federal Registration of Securities implies that loan-based crowdfunding should be exempt from any additional state-level registration requirements to avoid the issue of duel authorization and reduce complexity (Huang and Zhao, 2017; Wolfe and Yoo, 2017).

\section{Fundraising and Investment Limits}

According to fundraising regulations and investment limits, a fundraiser may raise capital to a specific amount of funds. Moreover, investment is capped for each investor in crowdfunding through funding portals. The online nature of crowdfunding can add a new dimension to traditional issues with investor protection. As a consequence, it can affect the dynamics of the crowd as the decision to invest can be influenced by the online visibility of a campaign (Matthew, 
Table 7. Summary of fundraising and investment limit across countries

\begin{tabular}{|c|c|c|c|}
\hline \multirow{2}{*}{ Countries } & \multicolumn{2}{|c|}{ Fundraising limits over 12 months } & \multirow{2}{*}{ Securities } \\
\hline & Company & Investors & \\
\hline US & $\begin{array}{l}\text { Title III of the JOBS Act: } \\
\text { USD 1M } \\
\text { Title IV of the JOBS Act: } \\
\text { USD 20M (tier 1) and 50M (tier 2) }\end{array}$ & $\begin{array}{l}\text { Based on investor's annual income or net worth (Rui-Teng } \\
\text { Hsueh et al., 2016; Chang, 2020; Wroldsen, 2016; Mamonov and } \\
\text { Malaga, 2018): } \\
\text {-Less than USD 40K invest USD 2K } \\
\text {-Less than USD 100K invest 5\% } \\
\text {-More than USD 100K invest 10\% } \\
\text { Accredited investors (Rossi et al., 2019; Kourabas and Ramsay, } \\
\text { 2017; Wroldsen, 2016): No limit }\end{array}$ & Equity and Debt \\
\hline Italy & $\begin{array}{c}\text { EUR 5M and 5\% financed by accredited investors } \\
\text { (Macchiavello, 2018; Rossi et al., 2019) }\end{array}$ & No limit (Havrylchyk, 2018; Rossi et al., 2019) & Equity \\
\hline France & $\begin{array}{l}\text { EUR 1M (Gabison, 2015; Havrylchyk, 2018; Rossi et al., } \\
\text { 2019) }\end{array}$ & No limit (Havrylchyk, 2018; Rossi et al., 2019) & Equity and Debt \\
\hline Finland & No limit (Havrylchyk, 2018) & No limit (Havrylchyk, 2018; Rossi et al., 2019) & Debt \\
\hline UK & GBP 5M (Rossi et al., 2019) & $\begin{array}{c}\text { Non-accredited investors (Rossi et al., 2019): 10\% of net assets. } \\
\text { Accredited investors (Rossi et al., 2019; Kourabas and Ramsay, } \\
\text { 2017; Wroldsen, 2016): No limited }\end{array}$ & Equity and Debt \\
\hline Malaysia & $\begin{array}{l}\text { RM 3M with a total cap of RM 5M (Kourabas and Ramsay, } \\
\text { 2017) }\end{array}$ & $\begin{array}{l}\text { Retail investors (Kourabas and Ramsay, 2017): RM 5K per issuer } \\
\text { with a total amount up to RM 50K. } \\
\text { Angel investors: up to RM 500K } \\
\text { Accredited investors (Rossi et al., 2019; Kourabas and Ramsay, } \\
\text { 2017; Wroldsen, 2016): No limit }\end{array}$ & Equity \\
\hline Thailand & $\begin{array}{l}\text { THB 20M with a total cap THB 40M (Kourabas and } \\
\text { Ramsay, 2017) }\end{array}$ & $\begin{array}{l}\text { Retail investors (Kourabas and Ramsay, 2017): THB 50K per } \\
\text { issuer with a total of THB 500K. } \\
\text { Non-retail investors (Rossi et al., 2019; Kourabas and Ramsay, } \\
\text { 2017; Wroldsen, 2016): No limit }\end{array}$ & Equity \\
\hline Australia & AUD 5M (Rossi et al., 2019) & $\begin{array}{l}\text { Retail investors (Rossi et al., 2019; Kourabas and Ramsay, 2017): } \\
\text { AUD 10K per issuer Accredited investors (Rossi et al., 2019; } \\
\text { Kourabas and Ramsay, 2017; Wroldsen, 2016): No limit }\end{array}$ & Equity and Debt \\
\hline Singapore & $\begin{array}{l}\text { Less than SGD 5M of all offers without prospectus } \\
\text { (Hofmann, 2018) }\end{array}$ & & Equity \\
\hline Taiwan & $\begin{array}{l}\text { Less than TWD 15M if company capital is less than TWD } \\
\text { 30M (Rui-Teng Hsueh et al., 2016) }\end{array}$ & $\begin{array}{l}\text { Non-accredited investors (Rui-Teng Hsueh et al., 2016): up to } \\
\text { TWD 50K per project and up to TWD } 100 \mathrm{~K} \text { per platform }\end{array}$ & Equity \\
\hline Latvia & & $\begin{array}{l}\text { Non-accredited investors (Lukstinšs, 2017): } \\
\text { Up to EUR 10K to natural person, and EUR 100K to develop a } \\
\text { submitted project or real estate projects }\end{array}$ & Real state \\
\hline Spain & $\begin{array}{l}\text { EUR 2M or EUR 5M when directed to accredited investors } \\
\text { (Macchiavello, 2018) }\end{array}$ & $\begin{array}{c}\text { EUR 3K per issuer/ EUR 10K for all issues. Platform providers: } \\
\text { invest 10\% per loan (Macchiavello, 2018) }\end{array}$ & Equity and Debt \\
\hline Belgium & & EUR 5K invested by the general public (Havrylchyk, 2018) & Debt \\
\hline EU (in general) & EUR 1M (Havrylchyk, 2018; Rossi et al., 2019) & & Debt \\
\hline Canada & $\begin{array}{l}\text { (Start-up Exemption) law (Rossi et al., 2019; Huang and } \\
\text { Zhao, 2017): } \\
\text { CAD 250K with a maximum of } 2 \text { offerings. } \\
\text { (Integrated Crowdfunding Exemption) law (Huang } \\
\text { and Zhao, 2017; Kourabas and Ramsay, 2017; Rossi et al., } \\
\text { 2019): CAD 1.5M }\end{array}$ & $\begin{array}{c}\text { (Start-up Exemption) law: } \\
\text { CAD 1.5K per issuer. } \\
\text { (Integrated Crowdfunding Exemption) law: Non-accredited } \\
\text { investors (Havrylchyk, 2018; Kourabas and Ramsay, 2017; Rossi } \\
\text { et al., 2019): CAD 2.5K per issue, CAD 10K for all issues. }\end{array}$ & Equity and Debt \\
\hline Austria & EUR $1.5 \mathrm{M}$ & $\begin{array}{l}\text { Non-accredited investors: EUR 5K per issuer. } \\
\text { 10\% of the financial assets if investor monthly net income of the } \\
\text { least EUR } 2.5 \mathrm{~K} \\
\text { (Havrylchyk, 2018; Rossi et al., 2019) }\end{array}$ & Equity and Debt \\
\hline Germany & $\begin{array}{l}\text { EUR } 2.5 \mathrm{M} \\
\text { If exemption from the full prospectus requirement is } \\
\text { relied on. }\end{array}$ & $\begin{array}{c}\text { EUR } 1 \mathrm{~K} \text { per project without providing any statements, twice the } \\
\text { average monthly net income and up to EUR 10K with at least } \\
\text { EUR } 100 \mathrm{~K} \text { asset (Rossi et al., 2019) }\end{array}$ & Equity and Debt \\
\hline China & No more than 200 shareholders. & $\begin{array}{l}\text { Accredited investor: RMB } 1 \mathrm{M} \text { per project (Rui-Teng Hsueh et al., } \\
\text { 2016) }\end{array}$ & Equity \\
\hline New Zealand & NZD 2M (Kourabas and Ramsay, 2017) & No limit (Havrylchyk, 2018; Rossi et al., 2019) & Equity \\
\hline Netherland & EUR 40K (Huang and Zhao, 2017) & EUR 80K per project public (Macchiavello, 2018) & Debt \\
\hline Portugal & $\begin{array}{c}\text { EUR 1M per project and EUR 5M when directed to } \\
\text { accredited investors or individuals with an annual } \\
\text { income equal or higher than EUR 70K (Havrylchyk, 2018) }\end{array}$ & $\begin{array}{l}\text { EUR 3K per offer if annual income is less than EUR 70K with a } \\
\text { EUR 10K in total (Havrylchyk, 2018) }\end{array}$ & Debt \\
\hline Greece & EUR 500K (Spanos, 2018) & $\begin{array}{l}\text { EUR } 5 \mathrm{~K} \text { per project / EUR 30K in the same platform per year. } \\
\text { Should not exceed } 10 \% \text { of an investor's average income over the } \\
\text { past } 3 \text { years (Spanos, 2018) }\end{array}$ & Equity \\
\hline
\end{tabular}

2017). Investor protection across countries primarily highlights the importance of setting a limit on fundraising and investment as setting this limitation essentially supports the protection of a range of investors. Hence, it can have a significant impact on attracting potential investors. Table 7 represents the summary of fundraising and investment limits across countries.

\section{Investors (Funders)}

Finland, Italy, France and New Zealand regulatory authorities, unlike most other countries, do not restrict crowdfunding campaigns or investment limits for investors. For accredited investors, the US, Australia, Malaysia, Thailand and the UK specify no limits, whereas other countries as shown in Table 7 impose certain conditions. In some countries, the 
Table 8. Conditions, requirements and exemption from prospectus legislation in certain countries

\begin{tabular}{|c|c|c|c|}
\hline Countries & Prospectus conditions & Prospectus Requirement & $\begin{array}{l}\text { Prospectus Exemption } \\
\end{array}$ \\
\hline Italy & & & $\begin{array}{l}\text { For innovative start-ups, including start-ups with a social vocation } \\
\text { (Gabison, 2015) }\end{array}$ \\
\hline France & & & For fundraising up to EUR 1M (Gabison, 2015) \\
\hline Finland & & & $\begin{array}{l}\text { If the securities are offered in Finland and their combined } \\
\text { consideration over } 12 \text { months is less than EUR 5M (Havrylchyk, } \\
\text { 2018) }\end{array}$ \\
\hline UK & & & $\begin{array}{l}\text { Only for offers to the public of over GBP 5M, involves fewer } 150 \\
\text { non-qualified investors and any number of qualifies investors or for } \\
\text { investment that not exceed GBP 100K (Rui-Teng Hsueh et al., 2016) }\end{array}$ \\
\hline Thailand & & & $\begin{array}{l}\text { If the value of the securities being offered to retail investors does } \\
\text { not exceed THB 20M during a 12-month period (Kourabas and } \\
\text { Ramsay, 2017) }\end{array}$ \\
\hline Australia & & $\begin{array}{l}\text { Information statement and } \\
\text { profile statement (Gabison, } \\
\text { 2015) }\end{array}$ & \\
\hline Singapore & & & $\begin{array}{l}\text { For small offers when the total amount raised from all offers by the } \\
\text { issuer of securities within a year does not exceed the amount of } \\
\text { SGD 5M, private placements, and offers to institutional investors or } \\
\text { accredited investors (Hofmann, 2018) }\end{array}$ \\
\hline Latvia & $\begin{array}{l}\text { Require Financial and } \\
\text { Capital Market Commission } \\
\text { of Latvia (FCMC) to prepare } \\
\text { a prospectus, if the capital } \\
\text { raise exceeds GBP 100K } \\
\text { (Havrylchyk, 2018; } \\
\text { Lukstinš, 2017) }\end{array}$ & & \\
\hline Belgium & $\begin{array}{l}\text { Only for offers of GBP 100K } \\
\text { or more (Havrylchyk, 2018) }\end{array}$ & & $\begin{array}{l}\text { For offers below GBP 300K submitted under some conditions } \\
\text { (Havrylchyk, 2018) }\end{array}$ \\
\hline EU & $\begin{array}{l}\text { For offers of securities to } \\
\text { the public above EUR 8M } \\
\text { (Hofmann, 2018) }\end{array}$ & & $\begin{array}{c}\text { For offers with a total consideration of less than EUR 1M for all } \\
\text { offers in between EUR 1M and EUR 8M; member states are free in } \\
\text { their decision whether to require a prospectus in their national } \\
\text { jurisdictions (Hofmann, 2018) }\end{array}$ \\
\hline Canada & & & Start-ups and early stage companies (Kourabas and Ramsay, 2017). \\
\hline Germany & & & $\begin{array}{l}\text { If financing amount does not exceed EUR 2.5M (Havrylchyk, 2018; } \\
\text { Huang and Zhao, 2017) }\end{array}$ \\
\hline
\end{tabular}

regulators put investment limitations on crowdfunding campaigns based on the investors' annual income or net worth such as the US, Austria, Germany, Greece, and Portugal.

\section{Companies (Fund seekers)}

Crowdfunding regulatory authorities have imposed additional fundraising limits or offering limits on the amount of funds companies can raise through crowdfunding platforms. As shown in Table 7, companies (fund seekers) seeking investment have diverse limitations on how they raise funds in various countries such as New Zealand, Australia, Netherlands, Greece, France, Austria, EU in general, UK, Thailand and Malaysia. Regulators in certain countries have set a limit for issuers based on whether the funds are sought from accredited investors or general public investors such as Portugal, Italy, Spain, China, and Taiwan. A few countries have regulations on the offering limits for companies based on their prospectus, such as Germany and Singapore (Hofmann, 2018). Meanwhile, Finland has not set any fundraising limit on an issuer who is seeking funds from investors (Havrylchyk, 2018).

\section{Disclosures Obligations}

Companies (fund seekers) that are seeking funds through crowdfunding are usually required to produce disclosure documents. In this paper, disclosure documents were analyzed under four categories: the prospectus, financial information, annual report, and other forms of disclosures.

A prospectus must be attached to each security offer. Most of the countries are required to submit a prospectus, but some countries have extra conditions, additional requirements, and exemptions. Table 8 summarises the disclosure conditions of various countries. The EU, Belgium, and Latvia have set additional conditions along with prospectus obligation. Despite the existence of a large number of conditions along with prospectus obligation, Australia has additional requirements regarding the prospectus. The additional requirements are the information statement and profile statement (Gabison, 2015). In contrast, a number of countries have eliminated the prospectus requirement with exemptions such as Canada, the UK, Italy, Finland, France, Belgium, Thailand, Singapore and Germany, and members of the EU.

A financial statement is a compulsory disclosures obligation in many countries such as US, Canada, UK, Austria, Estonia, Portugal, Malaysia, Singapore, Australia and New Zealand, and members of the EU. Furthermore, the US and Estonia have issued additional conditions that accompany financial statement disclosures. In the US, entrepreneurs and startups must provide financial disclosures at three different thresholds: issuers offering USD 100,000 or less, issuers offering more than USD 100,000, but less than USD 500,000, 
Table 9. Examples of other forms of disclosures

\begin{tabular}{cc}
\hline $\begin{array}{c}\text { Region } \\
\text { US }\end{array}$ & Examples of other forms of disclosures \\
\hline France & A description of the ownership and capital structure of the issuer (Schwartz, 2020). \\
\hline Finland & General terms and conditions to users (Havrylchyk, 2018). \\
\hline UK & Providing product information to clients (COBS, 2020) \\
\hline Thailand & Disclosure of information regarding the issue of shares through the equity crowdfunding platform(Havrylchyk, 2018). \\
\hline Australia & Risk statement (Matthew, 2017) \\
\hline Singapore & Risk disclosure statement' that needs to be signed by every investor (Hofmann, 2018). \\
\hline Belgium & Description of the cost of the service (Havrylchyk, 2018). \\
\hline EU & Marketing communications about the costs and charges related to crowdfunding services or investments (Kourabas and \\
\hline Canada & Ramsay, 2017). \\
\hline Austria & Annual disclosure of information about the use of funds (Kourabas and Ramsay, 2017). \\
\hline Germany & All beneficial owners involved with at least 25\% (Havrylchyk, 2018) \\
\hline Mexico & Investment information sheet (Havrylchyk, 2018). \\
\hline Spain & Inform potential clients of the risks associated with their business model (Havrylchyk, 2018). \\
\hline Estonia & Policy on conflicts of interest and annual obligation to report to the supervisor (Macchiavello, 2018b) \\
\hline Malaysia & Disclose the information about if a crowdfunding platform operator does invest in an issuer (Kourabas and Ramsay, 2017). \\
\hline New Zealand & A statement that tells investors how the service works and the fees they will pay (Kourabas and Ramsay, 2017). \\
\hline Portugal & The description of the activity or product to be financed (Havrylchyk, 2018).
\end{tabular}

and issuers offering more than USD 500,000 (Groshoff et al., 2015). The Financial Supervision Authority of Estonia (FSA) requires an issuer to publish a financial report on the funding portal no later than two months after the end of each year (Lukstiňš, 2017).

Annual reports are forms of disclosures ordinance that are requested by some countries such as the US, Canada, Spain, Austria, France and Portugal. In the US, an annual report is a mandatory disclosure in company with additional conditions: it must be submitted each fiscal year to the Security Exchange Commission (SEC) and be published on the website (Groshoff et al., 2015).

The majority of the countries have requested some other forms of disclosure apart from the prospectus, financial information and annual report obligations. Table 9 contains a list of countries and their other forms of disclosures. More to the point, Australia has an exemption with the disclosure's obligation. Australia provides exemption on the disclosure's requirement with the following conditions:

- Small-scale offerings (Gabison, 2015; Kourabas and Ramsay, 2017)

- Securities offering involving 20 unaccredited investors and less than AUD 2 million valuations within the last twelve months (Gabison, 2015).

- Offerings limited to experienced investors within the issuer cap of AUD 5 million (Kourabas and Ramsay, 2017).

Startups or early-stage companies can be crowdfunded by investors by issuing securities through the prospectus exemption. Generally, an investment prospectus creates a significant cost, which may not pay off for the start-up and early-stage businesses. The primary reason is the prospectus must incorporate all necessary details and any mistake may lead to liability issues for the company, its directors, and underwriters in the prospectuses. Therefore, start-ups are interested in avoiding the prospectus requirements to as great an extent as possible (Hofmann, 2018; Kourabas and Ramsay, 2017; Lukstiņš, 2017). Moreover, the cost of generating crowdfunding financial statements and annual reports is often a major expense in any securities offering as well as an obstacle when start-ups and early-stage businesses try to raise capital. On the other hand, making financial disclosure and annual reports available to potential investors could help them to make a better investment decision (Groshoff et al., 2015). The investors can analyze the information provided through disclosure and assess the company to determine whether it is suitable for them to invest. Better disclosure of information can also assist in avoiding the risk of potential fraud. Even though disclosure requirements help the investor to evaluate the issuer, admittedly they are an insufficient pre-condition for the greatest issuer protection from the perspective of startup and new business (Gabison, 2015; Kourabas and Ramsay, 2017; Mills, 2016).

\section{Crowdfunding Advertising Law}

Most of the literature suggested compelling existence of crowdfunding advertising obligations on crowdfunding platform operators and issuers. Countries such as Singapore, Malaysia, Spain and Canada have imposed restrictions that prohibit the advertisement of offers. For example, in Canada, the issuers are restricted in promoting their offers other than to refer prospective investors to the offering document on the ECF platform. In contrast, a number of exemptions are provided in some countries, such as the UK, the US, and Thailand, that allow crowdfunding platform operators and issuers to advertise.

In the UK, advertising is allowed for a restricted number of investors such as professional clients and sophisticated or high net worth retail clients, to name a few. The US allows accredited investors through intermediaries, and Thailand allows platforms to advertise provided that advertising is relevant and accurate (Kourabas and Ramsay, 2017). Advertisement is the best tool to create publicity that will be an important resource for issuers and platforms in order to gain more investors (Gabison, 2015). Allowing common solicitation or advertising of offerings might create a considerable legal space in fundraising transactions (Huang 
Table 10. Capital requirements regulations across countries

\begin{tabular}{|c|c|}
\hline Region & Capital Requirements regulations \\
\hline Belgium & $\begin{array}{l}\text { No minimum capital requirement, professional liability insurance of at least EUR 750K per claim and insurance year; this amount } \\
\text { increases to EUR } 1.25 \text { million when investment advice is provided or when instruments are issued by an investment vehicle } \\
\text { (Havrylchyk, 2018) }\end{array}$ \\
\hline Germany & $\begin{array}{c}\text { No minimum capital requirement; platform should have a commercial license and the minimum sum insured amounts to EUR } \\
1,276,000 \text { per insured event and to EUR } 1,919,000 \text { in regards to all insured events in one year (Havrylchyk, 2018) }\end{array}$ \\
\hline Finland & $\begin{array}{l}\text { EUR 50K in equity or professional liability insurance policy, bank guarantee or other any other document that is deemed to be } \\
\text { sufficient by the Financial Supervisory Authority (FSA) (Havrylchyk, 2018) }\end{array}$ \\
\hline Taiwan & Platform should have capital of more than TWD 50M while companies must have less than TWD 30M (Rui-Teng Hsueh et al., 2016) \\
\hline Spain & EUR 60K: that value can increase depending on the volume of transactions (Herrera, 2016; Macchiavello, 2018) \\
\hline France & No minimum capital requirement, a professional liability insurance policy covering EUR 250,000 per event (Havrylchyk, 2018) \\
\hline Latvia & EUR 50K (Havrylchyk, 2018; Lukstin̦š, 2017; Macchiavello, 2018) \\
\hline EU & $\begin{array}{l}\text { EUR 730K set by Markets in the Financial Instruments Directive (MiFID) but at EUR 125K where a firm does not deal on its own } \\
\text { account (Ahern, 2018) }\end{array}$ \\
\hline UK & $\begin{array}{l}\text { GBP } 50 \mathrm{~K} \text { or it is expressed in terms of percentage of the loan requested }(0.2 \% \text { of the first GBP } 50 \mathrm{M} \text { of that total value, } 0.15 \% \text { of the } \\
\text { next GBP } 200 \mathrm{M} \text { of that total value, } 0.1 \% \text { of the next GBP } 250 \mathrm{M} \text { of that total value and } 0.05 \% \text { of any remaining total value) (Ahern, } \\
\text { 2018; Havrylchyk, 2018; Hofmann, 2018) }\end{array}$ \\
\hline Portugal & $\begin{array}{l}\text { EUR 50K requirement or have liability insurance policy or mix of both that grants the investors the same type of protection and is } \\
\text { accepted by the Portuguese Securities Market Commission (CMVM) (Havrylchyk, 2018; Lukstinš, 2017; Macchiavello, 2018) }\end{array}$ \\
\hline Singapore & Monetary Authority of Singapore (MAS) has set a minimum capital requirement of SGD 50,000 (Kourabas and Ramsay, 2017) \\
\hline
\end{tabular}

Table 11. Summary of clients' fund policy across the country

\begin{tabular}{|c|c|}
\hline $\begin{array}{l}\text { Region / } \\
\text { Countries }\end{array}$ & Clients' funds policies \\
\hline Austria & Should rely on the payment institution (Havrylchyk, 2018) \\
\hline Belgium & Platforms cannot hold clients’ funds (Havrylchyk, 2018) \\
\hline Mexico & Keep their clients' deposits separated from their own resources (Havrylchyk, 2018) \\
\hline Finland & $\begin{array}{l}\text { The platform has to rely on banking services (licensed) or payment institutions or they should apply for registration as a payment } \\
\text { institution (Havrylchyk, 2018) }\end{array}$ \\
\hline France & No platforms can receive any money unless they obtain the status of agent providing payment services (Havrylchyk, 2018) \\
\hline Latvia & Platforms must ensure that the money received from investors is stored in a separate account (Lukstinšs, 2017) \\
\hline $\mathrm{EU}$ & $\begin{array}{c}\text { According to Article } 4 \text { (11) of Directive (EU) 2015/2366, platforms do not keep the lent money and require appropriate segregation } \\
\text { of investors' and borrowers' monies from that of the platform operator. }\end{array}$ \\
\hline UK & $\begin{array}{l}\text { Platforms must ensure that the client's money is kept separate from the platform's own funds, kept with the institutions (must be } \\
\text { a bank), and the bank must acknowledge that the money in the account is held for the clients of the firm and that the bank cannot } \\
\text { recover the debts of the firm from those accounts (COBS, 2020). }\end{array}$ \\
\hline Singapore & $\begin{array}{l}\text { Place funds into trust accounts and assets into custody accounts, prohibited from holding any clients' monies in their own } \\
\text { accounts (Hofmann, 2018). }\end{array}$ \\
\hline
\end{tabular}

and Zhao, 2017). Under the consideration of consumer protection and advertising, it is better to have an option of a "cooling-off" period that may attract more potential investors to make an investment decision. In the meantime, they also will be able to reconsider or annul their decision (Lukstiņš, 2017).

\section{Capital Requirements Policy}

Under the crowdfunding regulatory framework, the platform operators and issuers are required to comply with the minimum capital requirement. In most countries, funding portal operators should have minimum capital requirements to operate or should have a professional liability insurance policy. Table 10 summarizes the capital requirements regulations across countries and the conditions. Several studies have concluded that providing capital requirements regulations and directives will deliver a degree of protection for investors in the event of platform portal insolvency. Also, it would have the additional advantage of operational continuity in mitigating portal closure risks. Moreover, this regulation would help in aligning incentives between portal shareholders and investors (Ahern, 2018; Havrylchyk, 2018; Monés, 2018).

\section{Protection of Clients' Funds}

Regarding the management of investors' funds, regulatory authorities have set rules on how investors' money is to be handled. Overall, most of the countries agreed that platforms should maintain a separate account for clients' money from the platforms' fund. Table 11 summarizes the client fund policy of various countries. For example, Belgium denotes that "Platforms cannot hold client's funds," and Mexico orders platforms to "keep their clients' deposits separated from their own resources" (Havrylchyk, 2018). Latvia also has strict rules, "ensuring that the money received from investors is stored in a separate account” (Lukstiņš, 2017).

Moreover, there are pre-conditions established when it comes to protecting clients' fund. The pre-conditions maintain two separate accounts for clients' funds and 
platform's operating account aims to protect investors from any mishandling of their money by the platform. This precondition helps minimize losses in the case of insolvency. While being conscious of investor protection, the regulation code of conduct also set out the rules in the distribution of repayment. This regulation would serve as a measure of antimoney laundering that complies with the best practice of gathering accurate personal information and banking details and pays attention to suspicious money laundering activities (Herrera, 2016; Hofmann, 2018; Lukstiņš, 2017).

\section{Platform-Specific Regulation}

Despite the investor's perspective, the US has added a special regulation by the SEC based on the perspective of the recipient of equity crowdfunding. The act pinpoints the national student debt dilemma and introduces a method that is based on "Human Capital Contract" (HCC). HCC funding platforms allow students accepted at an institution to have higher education, as they will finance the cost of education. This type of platform permits the human capital investor to obtain a share in the graduate's future income by making a contribution to the graduate's education. HCC develops a legal framework that initiates a repayment scheme with a specific period which starts after a student's graduation while also ensuring the repayment is exempted from bankruptcy discharge. Furthermore, HCC funds are essentially taxable (Groshoff et al., 2015). HCC revolutionizes the way the crowdfunding works. These contracts represent an economically-feasible, socially-responsible and noninstitutionalized framework that postulates higher education as an investment in human capital (Groshoff et al., 2015). HCC funding platforms enable graduates to pursue their higher education as a means of financing by resolving their debt burdens. HCC crowdfunding portals enable financial risk transfer from the graduate to lenders and could help combat the US student community loan problem. Moreover, the graduates raising funds are incentivized in order to promote the platform to their community as they can raise debt-free funds from diverse investors.

Greece has set regulations for non-financial return platforms, more specifically for donation-based crowdfunding activity. In Greece, this regulation required fundraisers to obtain a special license from the Ministry of Labor and Social Solidarity. Hence, non-profit entities (NGOs) are able to initiate fundraising or donation activities. According to the law 5101/1931 that was amended in December 2015, fundraising and donation activities could only be initiated by a banking institution through an online platform (Spanos, 2018). Australia has enacted a special Act (the Competition and Consumer Act 2010) under Australian Consumer law, which captures companies that engage in a pre-ordering reward-based crowdfunding. Furthermore, the law protects consumers from misleading information about goods and services. The underlying reason is that when the donor merely makes a contribution, reward-based crowdfunding is unlikely to fall within the guidelines of any current consumer protection legislation (Matthew, 2017). The number of laws on non-financial return crowdfunding that could prevent illegal fundraising activities through online platforms has immensely increased in recent years. Also, these laws could act as a proactive solution to money-laundering. Moreover, the special license provides a legal reference for non-profit entities to have fundraising activities that serve a philanthropic purpose or utilities. Figure 1 summarizes classified regulations themes against the selected countries.

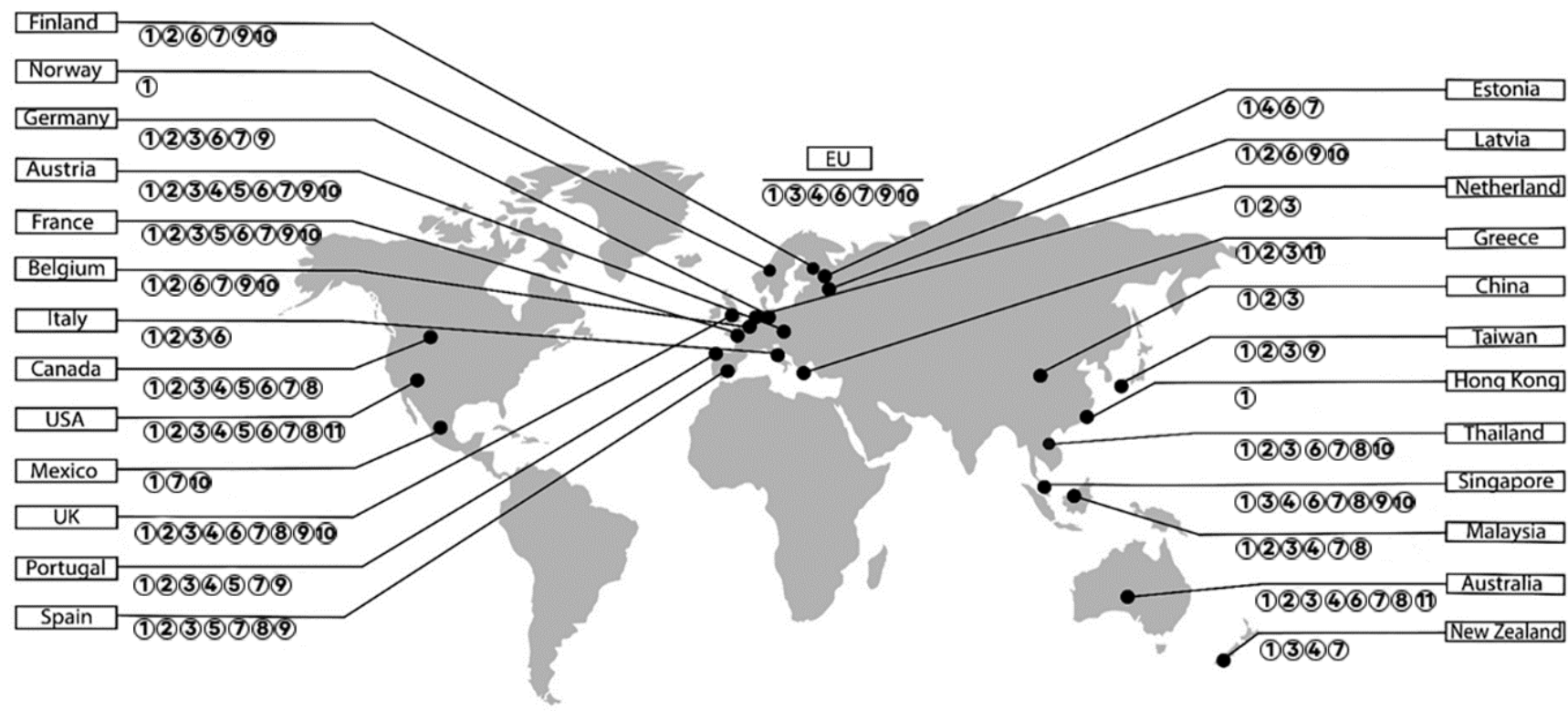

(1) Authorization (2) Investor's Limit (3)Company's Limit

(4) Financial Disclosures (5) Annual Report Disclosures (6) Prospectus

(7) Other Forms of Disclosures (8) CF Advertising Law (9) Capital Requirements Policies (10) Protection of Client's Fund (11) Platform Specific Regulation

Figure 1. Classified regulation requirements against selected countries 


\section{DISCUSSIONS}

This paper has identified the key regulatory requirements based on the systematic literature review of countries crowdfunding regulatory acts. Through thematic analysis, the regulation requirements are authorization, fundraising and investment limits, disclosures obligations, crowdfunding advertising law, capital requirements policies, protection of client funds, and platform-specific regulation. These regulation requirements contribute to developing a generic crowdfunding regulatory framework. The study suggests that the framework must focus on the regulations that ensure investor protection primarily while managing the risk of the platform's insolvency or embezzlement.

The study found that authorization regulation is a significant requirement in the crowdfunding process. The authorization ensures the platforms comply with legal regulations and reflect high standards of commercial integrity as well as refrain from fraudulent activities. Fundraising and investment limits are another important requirement that mainly focuses on investor protection, which affects the "dynamics of the crowd". The exemption on disclosures obligations will enable the start-ups and early-stage companies to make easy registration and help them engage in jurisdictions to raise capital. Moreover, with the disclosure's requirements, investors can make a better-informed investment decision and avoid potential fraud. While crowdfunding advertising law helps to attract more investors, capital requirement policies and protection of client funds deliver a degree of protection for investors in the case of insolvency. Non-financial return platforms in Greece and Australia are mainly built on protecting individuals from illegal fundraising, and money-laundering activities. Platform-specific requirements such the US-bound HCC funding portals allow the graduate to have higher education which primarily motivates community development.

Additionally, this study suggests that policymakers must consider light-touch regulations that can benefit borrowers. It is also important to note that exemptions appear to have gained prominence among startups and early-stage companies. The study also presented evidence that regulations must certify the actors (intermediaries, investors, and entrepreneurs) involved in the crowdfunding landscape have met the certain threshold requirements. The formulated regulations must also guarantee compliance with organizational requirements.

This study benefits primarily four parties such as the government, companies (fund-seekers), investors (funders), and the platform owners from the practical perspective. For government, this study suggests a formation of a centralized authority in monitoring various crowdfunding industry projects can establish new communication channels with the companies. This government support system will cope with high demand from the companies. Also, the government must create advertisement policies that will enable more companies to engage in crowdfunding. The legislation structure must be revamped, based on domestic situations as well as analyzed and formulated by comparing foreign cases. For companies, this study enables them to understand the existing regulation risks and security measures. They could then establish a standard in order to overhaul existing security issues. For investors, this study helps them to keep updated on regulations and legislation changes before committing to any investment. Also, this study enables investors to make better investment decisions by examining the crowdfunding regulatory requirements.

For platform owners, this study suggests that the incentive schemes in case of start-up failures should be built into the platform so that they can maintain competitiveness among their competitors (Ibrahim, 2016). When developing the regulatory features in the platform particularly for lendingbased, Mills (2016) proposed the Regulatory Action Plan (RAP), which promotes small business lending space by creating new rules for the protection of the small business owner. Borrowers protection can be achieved by clear and concise disclosures requirements that allow borrowers to determine whether they will be able to comply with the terms and conditions of the raised capital.

Moreover, the disclosures requirements suggested in this study can be further enhanced by the establishment of a National Advisory Board to implement a more coordinated regulatory approach on financial innovation. This authority will combine major government regulators that have preview in lending, borrower protection advocates, and FinTech executives. They will be tasked with advising policymakers on the regulatory structure. Moreover, this body will involve actively taking advantage of technological innovation in order to execute a regulatory task efficiently. This approach can be achieved by incorporating specific models such as "innovation boxes," “sprints," etc.

\section{CONCLUSION AND FUTURE WORKS}

In summary, this study enables underdeveloped or developing countries to understand the crowdfunding regulatory requirements better by offering them the fundamental regulatory knowledge. Echoing Frydrych and Kinder (2015), this study emphasizes the development of entrepreneurial culture by educating market participants such as the entrepreneurs and investors in these nations where they see alternative financial technologies as threats rather than opportunities.

As for future work, the identified regulation requirements can serve as a generic regulatory framework and can be futher extended in the future from several perspectives. From the theoretical perspective, scholars can further explore the feasibility of adopting other social or cultural theories in solidifying the regulatory framework. Socio-cultural factors are essential when determining policy in order to ensure it is fit for its purpose. The generic regulatory framework could be evaluated and adapted in selected countries to ensure its applicability in the country specific regulatory systems by considering culture and constitutional differences.

Moreover, scholars could apply this generic regulatory framework to derive specific legal requirements for a specific crowdfunding type/platform. Scholars could also compare and contrast the regulation requirements not only based on different countries, but also how they work in different 
crowdfunding platform types. The regulatory framework could also be customised based on country-specific requirement and used for deriving guidelines in monitoring crowdfunding activities.

Author contributions: All co-authors have involved in all stages of this study while preparing the final version. They all agree with the results and conclusions.

Funding: No external funding is received for this article.

Declaration of interest: The authors declare that they have no competing interests.

Ethics approval and consent to participate: Not applicable. Availability of data and materials: All data generated or analyzed during this study are available for sharing when appropriate request is directed to corresponding author.

\section{REFERENCES}

Ahern, D. M. (2018). Regulatory arbitrage in a FinTech World: Devising an optimal EU regulatory response to crowdlending. SSRN Electronic Journal. https://doi.org/10.2139/ssrn.3163728

Ahlstrom, D., Cumming, D. J. and Vismara, S. (2018). New methods of entrepreneurial firm financing: Fintech, crowdfunding and corporate governance implications. Corporate Governance: An International Review, 26(5), 310313. https://doi.org/10.1111/corg. 12258

Aisyah, M. (2018). Islamic bank service quality and its impact on Indonesian customers' satisfaction and loyalty. AlIqtishad: Jurnal Ilmu Ekonomi Syariah, 10(2), 367-388. https://doi.org/10.15408/aiq.v10i2.7135

Al-Emran, M., Mezhuyev, V. and Kamaludin, A. (2018). Technology acceptance model in m-learning context: A systematic review. Computers \& Education, 125, 389-412. https://doi.org/10.1016/j.compedu.2018.06.008

Chang, J.-W. (2020). The economics of crowdfunding. American Economic Journal: Microeconomics, 12(2), 46. https://doi.org/10.1257/mic.20170183

Chowdhury, F., Audretsch, D. B. and Belitski, M. (2019). Institutions and entrepreneurship quality. Entrepreneurship Theory and Practice, 43(1), 51-81. https://doi.org/10.1177/1042258718780431

COBS. (2020). FCA Handbook, government. Available at: www.handbook.fca.org.uk

Estrin, S., Gozman, D. and Khavul, S. (2016). Case study of the equity crowdfunding landscape in London: An entrepreneurial and regulatory perspective. https://doi.org/10.5465/ ambpp.2016.13036abstract

Fenwick, M., McCahery, J. A. and Vermeulen, E. P. M. (2017). Fintech and the financing of entrepreneurs: From crowdfunding to marketplace lending. SSRN Electronic Journal. https://doi.org/10.2139/ssrn.2967891

Frydrych, D. and Kinder, T. (2015). How new is crowdfunding? The venture capital evolution without revolution Discourse on risk capital themes and their relevance to Poland. Problemy Zarzadzania, 13(56), 177-194. https://doi.org/10.7172/1644-9584.56.11
Gabison, G. A. (2015). Equity crowdfunding: All regulated but not equal. DePaul Business \& Commercial Law Journal, 13(3), 53. Available at: https://via.library.depaul.edu/bclj/ vol13/iss $3 / 2$

Groshoff, D., Nguyen, A. and Urien, K. (2015). Crowdfunding 6.0: Does the SEC's FinTech law failure reveal the agency's true mission to protect - Solely Accredited - Investors. Ohio State Entrepreneurial Business Law Journal, 9(2), 277321. https://doi.org/10.2139/ssrn.2483371

Havrylchyk, O. (2018). Regulatory framework for the loan-based crowdfunding platforms. OECD.

Herrera, D. (2016). Alternative finance (crowdfunding) regulation in Latin America and the Caribbean: A balancing act. InterAmerican Development Bank. https://doi.org/10.18235/ 0000430

Hofmann, C. (2018). An easy start for start-ups: Crowdfunding regulation in Singapore. Berkeley Business Law Journal, 15(1), 219-267.

Hsueh, R.-T., Lin, K.-C., Shyu, J. Z. and Li, K.-P. (2016). Equity crowdfunding: A new social innovation-a regulatory crossnation study. 2016 Portland International Conference on Management of Engineering and Technology (PICMET), presented at the 2016 Portland International Conference on Management of Engineering and Technology (PICMET), IEEE, Honolulu, HI, USA, pp. 1346-1350. https://doi.org/10.1109/PICMET.2016.7806539

Huang, T. and Zhao, Y. (2017). Revolution of securities law in the Internet Age: A review on equity crowd-funding. Computer Law \& Security Review, 33(6), 802-810. https://doi.org/10.1016/j.clsr.2017.05.016

Ibrahim, D. M. (2016). Crowdfunding without the crowd. North Carolina Law Review, 95, 1481-1506. Available at: https://ssrn.com/abstract=2854347

Jagtiani, J. and Lemieux, C. (2018). Do fintech lenders penetrate areas that are underserved by traditional banks? Journal of Economics and Business, 100, 43-54. https://doi.org/10.1016/j.jeconbus.2018.03.001

Kabai, M. (2017). Twist and turns of crowdfunding regulation. Journal of Public Administration, Finance and Law, 11, 182189.

Kitchenham, B. (2004). Procedures for performing systematic reviews. Keele University Technical Report TR/SE-0401.

Kitchenham, B. (2007). Guidelines for performing systematic literature reviews in software engineering. EBSE Technical Report EBSE-2007-01.

Kourabas, S. and Ramsay, I. (2017). Facilitating equity crowdfunding in the ASEAN Region. Association of Southeast Asian Nations (ASEAN). Available at: https://ssrn.com/abstract=3126652

Lee, T. and Kim, H.-W. (2015). An exploratory study on fintech industry in Korea: Crowdfunding case. 2nd International Conference on Innovative Engineering Technologies (ICIET'2015). Bangkok. https://doi.org/10.15242/IIE. E0815045

Lukstiņš, M. (2017). Real estate crowdfunding: Potential in Latvia. Riga Graduate School of Law. Available at: https://dspace.lu.lv/dspace/handle/7/45357 
Macchiavello, E. (2018). Financial-return crowdfunding and regulatory approaches in the shadow banking, FinTech and collaborative finance era. European Company and Financial Law Review, 14(4), 662-722. https://doi.org/10.1515/ecfr2017-0030

Maguire, M. and Delahunt, B. (2017). Doing a thematic analysis: A practical, step-by-step guide for learning and teaching scholars. All Ireland Journal of Teaching and Learning in Higher Education, 8(3), 3351-3364. Available at: http://ojs.aishe.org/index.php/aishe-j/article/view/335

Mamonov, S. and Malaga, R. (2018). Success factors in Title III equity crowdfunding in the United States. Electronic Commerce Research and Applications, 27, 65-73. https://doi.org/10.1016/j.elerap.2017.12.001

Matthew, A. (2017). Crowd-sourced equity funding: The regulatory challenges of innovative fintech and fundraising. University of Queensland Law Journal, 36(1), 41-71. https://search.informit.org/doi/10.3316/agispt. 20173758

Milian, E. Z., Spinola, M. de M. and Carvalho, M. M. de. (2019). Fintechs: A literature review and research agenda. Electronic Commerce Research and Applications, 34, 100833. https://doi.org/10.1016/j.elerap.2019.100833

Mills, K. (2016). The state of small business lending: Innovation and technology and the implications for regulation. SSRN Electronic Journal. https://doi.org/10.2139/ssrn.2877201

Monés, A. V. (2018). Regulation of crowdlending in the European Union. Understanding the suitableness of EU legislation for crowdlending (Thesis - LL.M International Business Law), Tilburg University.

Ng, A. W. and Kwok, B. K. (2017). Emergence of fintech and cybersecurity in a global financial centre. Journal of Financial Regulation and Compliance, 25(4), 422-434. https://doi.org/10.1108/JFRC-01-2017-0013
Rau, R. (2020). Law, trust, and the development of crowdfunding. SSRN Electronic Journal. https://doi.org/10.2139/ssrn.2989056

Regulatory Framework for the Loan-Based Crowdfunding Platforms, OECD Economics Department Working Papers No. 1513. (2018). https://doi.org/10.1787/24ad924a-en

Rossi, A., Vismara, S. and Meoli, M. (2019). Voting rights delivery in investment-based crowdfunding: a crossplatform analysis. Journal of Industrial and Business Economics, 46(2), 251-281. https://doi.org/10.1007/s40812018-0109-X

Sahaym, A., Datta, A. A. and Brooks, S. (2019). Crowdfunding success through social media: Going beyond entrepreneurial orientation in the context of small and medium-sized enterprises. Journal of Business Research, 125,483-494. https://doi.org/10.1016/j.jbusres.2019.09.026

Schwartz, A. A. (2020). Crowdfunding issuers in the United States. Washington University Journal of Law \& Policy, 61, 155.

Setälä, K. (2017). Crowdfunding in the banking industry: Adjusting to a digital era (Master's thesis), Jyväskylä University.

Spanos, L. (2018). Complementarity and interconnection between CSR and crowdfunding: A case study in Greece. In Corporate responsibility and digital communities (pp. 29-49). Palgrave Macmillan, Cham. https://doi.org/10.1007/978-3319-63480-7_3

Wolfe, B. and Yoo, W. (2017). Crowding out banks: Credit substitution by peer-to-peer lending. SSRN Electronic Journal. https://doi.org/10.2139/ssrn.3000593

Wroldsen, J. (2016). Crowdfunding investment contracts. 11 Virginia Law \& Business Review, 543, 75. Available at: https://ssrn.com/abstract=2844771 